\title{
Transducer Module Development for an Open-Source Ultrasound Tomography System
}

\author{
Morgan Roberts*, Eleanor Martin, Michael Brown, Ben Cox, Bradley Treeby \\ Department of Medical Physics and Biomedical Engineering, University College London, UK \\ *Email: morgan.roberts.18@ucl.ac.uk
}

\begin{abstract}
Researchers in the Ultrasound Tomography community face a high barrier to entry, due to the high cost and lead times associated with custom transducer arrays. For this work, 16-element linear array transducer modules have been developed for an open-source, low-cost, ring array with low interelement variation. A module prototype was characterised using a hydrophone to measure the pressure field from each element, and the transmit frequency response. The measured average elevation and lateral plane skews were $0.41^{\circ}$ and $2.07^{\circ}$, the elevation beamwidth was $8.1 \mathrm{~mm} \pm 0.23 \mathrm{~mm}$, and after normalisation the standard deviation in transmit amplitude spectrum between elements was $8 \%$, averaged across all frequencies. The low interelement variation in these parameters suggests that an ideal transducer model could be used to represent the elements during image reconstruction, which could be based on measurements from only a subset of elements, simplifying preliminary UST work for end users.
\end{abstract}

Index Terms-tomography, transducer, open-source, low-cost

\section{INTRODUCTION}

Mammographic breast cancer screening extends lives, but false positive and over-diagnosed results can damage the wellbeing of previously healthy women [1]. Ultrasound Tomography (UST) is non-ionising, requires no breast compression, and is promising since it can provide quantitative 3D maps of the sound speed [2] and acoustic absorption [3] of breast tissue, which could be used to make better treatment decisions. For widespread adoption of UST, faster methods that still provide high quality images are required. New methods must be validated experimentally, but there is a high barrier to entry, since UST hardware is not available off-the-shelf, and custom systems are expensive. A modular UST system that is manufactured in-house could make it easier to test new methods, without the expense and lead times associated with custom commercial transducers. Following earlier prototyping [4], this work details the further development of a 16-element transducer module for an open-source transducer system. It is designed to be low-cost, with fabrication processes that do not rely on specialist equipment, making it accessible to end users.

This work was supported by the National Physical Laboratory, European Union's Horizon 2020 research and innovation programme H2020 ICT 2016-2017 under Grant Agreement No. 732411 (as an initiative of the Photonics Public Private Partnership), the Engineering and Physical Sciences Research Council (EPSRC), UK, Grant Nos. EP/L020262/1, EP/S026371/1, and EP/P008860/1, and the EPSRC Centre For Doctoral Training in Intelligent Integrated Imaging In Healthcare (i4health).

\section{Design of a Modular UST System}

A modular research system can be reconfigured into various $2 \mathrm{D}$ and $3 \mathrm{D}$ apertures of different sizes, and is also easier to maintain, characterise and extend in future. Figure 1 shows the standard 2D ring array configuration for this system, which is approximated by a hexadecagon, constructed from 16 linear array modules, and has a diameter of $220 \mathrm{~mm}$. Arrays with dense spatial sampling are impractical to manufacture, and require a data acquisition system with thousands of channels or multiplexing capability. Instead the standard ring aperture for this system has only 256 elements, to match the number of channels available on common data acquisition platforms. Advanced UST systems rotate or translate arrays to access more spatial sampling positions [5], but static systems with 256 elements are still valuable for preliminary UST experiments [6]. Although 256 elements gives a sparse aperture, full waveform methods can be used to reconstruct high resolution images with fewer measurement positions than are required for ray-based tomography [7].
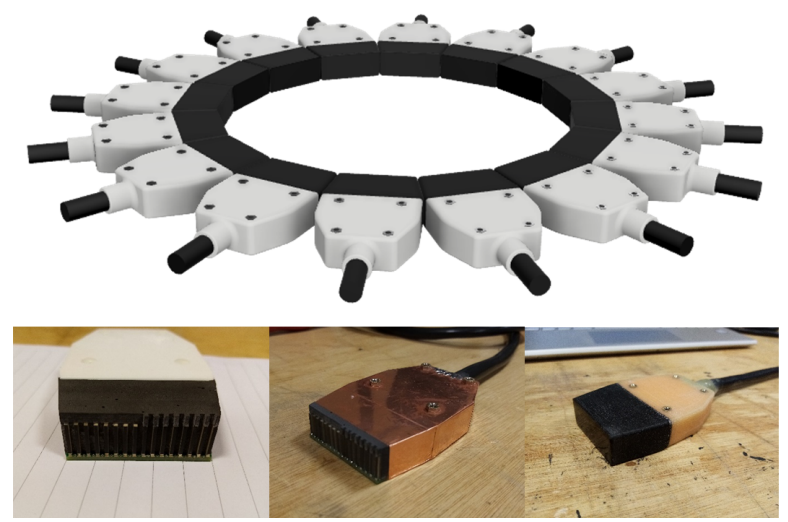

Fig. 1. Top: CAD mock-up of the standard ring aperture. Bottom: 16-element prototype transducer module at different assembly stages.

Linear arrays are typically made from diced PZT slabs, but this system uses off-the-shelf individual PZT bars as an accessible alternative. The PZT thickness is $1 \mathrm{~mm}$, giving a half-wavelength in-air resonance of $2 \mathrm{MHz}$. For 2D UST ring arrays, the beams are focused in the elevation plane and unfocused in the lateral plane. To select an element elevation height that gives a narrow beamwidth across the entire ring array, pressure fields from elements with varying heights were simulated across the $220 \mathrm{~mm}$ ring diameter, 
using the acoustic field propagator from the k-Wave toolbox [8]. The elements were represented as band-limited source distributions [9], on a $20 \times 140 \times 930$ grid, with a $0.25 \mathrm{~mm}$ step size, a uniform $1500 \mathrm{~m} / \mathrm{s}$ sound speed, a $2 \mathrm{MHz}$ source frequency, and a $1 \mathrm{~mm}$ element lateral width. The pressure on the element elevation plane was multiplied by a flipped copy of itself to find the transmit-receive sensitivity between two opposite elements, shown in Figure 2. The $-6 \mathrm{~dB}$ beamwidth of this sensitivity, as a function of axial position, is shown for each elevation height in Figure 2. A height of $10 \mathrm{~mm}$ was selected, since it gives a beamwidth less than $8 \mathrm{~mm}$ through the entire $140 \mathrm{~mm}$ region of interest. The beamwidth at the centre of the array is predicted to be $7.5 \mathrm{~mm}$. In the lateral plane, a $10 \mathrm{~mm} \times 1 \mathrm{~mm}$ element produces an opening angle of $50^{\circ}$. Although decreasing the element width would give a larger opening angle, wider elements have a larger area which provides a higher sensitivity, and they are easier to handle during assembly which may help to increase yields and decrease inter-element variation.

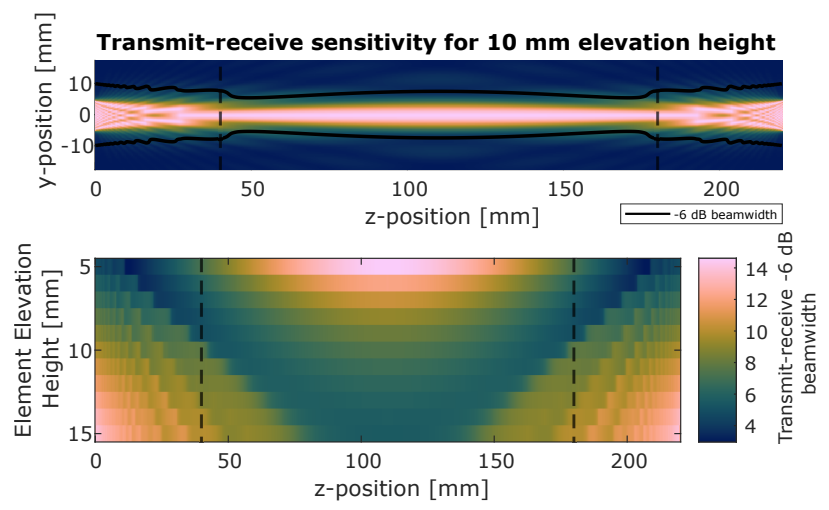

Fig. 2. Top: Transmit-receive sensitivity for an element with $10 \mathrm{~mm}$ elevation height (normalised in the y-direction at each axial z-position). Bottom: Dependence of axial beamwidth profile on element elevation height. A 140 $\mathrm{mm}$ region of interest, representing a breast, is highlighted with dashed lines.

The system uses a single tungsten-epoxy matching layer on the front of each PZT element. Araldite Standard epoxy resin (Huntsman) was chosen since it is cheap and its high viscosity prevents settling of tungsten particles. For a single matching layer, the required acoustic impedance is $Z_{m}=\sqrt{Z_{P Z T} Z_{w}}=$ $\sqrt{31 \times 1.5}=6.77$ MRayl. Acoustic property measurements performed in-house show that this impedance is provided by a composite with a $86.9 \%$ tungsten weight ratio, which has a sound speed of $1317 \mathrm{~m} / \mathrm{s}$, and requires a quarter wavelength matching layer thickness of $\lambda / 4=165 \mathrm{~mm}$ at $2 \mathrm{MHz}$.

For the backing layer, a tungsten weight ratio of $80.8 \%$ was used, which is estimated to provide an absorption coefficient of $\alpha \approx 7.8 \mathrm{~dB} / \mathrm{cm}$ at $0.6 \mathrm{MHz}$, using linear interpolation from [10]. This provides $37 \mathrm{~dB}$ of absorption across the $48 \mathrm{~mm}$ round-trip through the backing, at the lowest frequency component previously seen in the spectrum of internally reflected waves, measured during pulse-echo tests [4]. A scattering structure was used on the rear face of the backing layer with a wedge angle of $45^{\circ}$, to further increase attenuation in the backing layer, shown in Figure 3B.

\section{Assembly Methods}

A cross-sectional schematic of the transducer can be seen in Figure 3B. First, individual $10 \mathrm{~mm} \times 1 \mathrm{~mm} \times 1 \mathrm{~mm}$ PZT bars (American Piezo Ceramics) were placed in slots in a $3 \mathrm{D}$ printed polyvinyl alcohol (PVA) mould, with $1.165 \mathrm{~mm}$ depth.

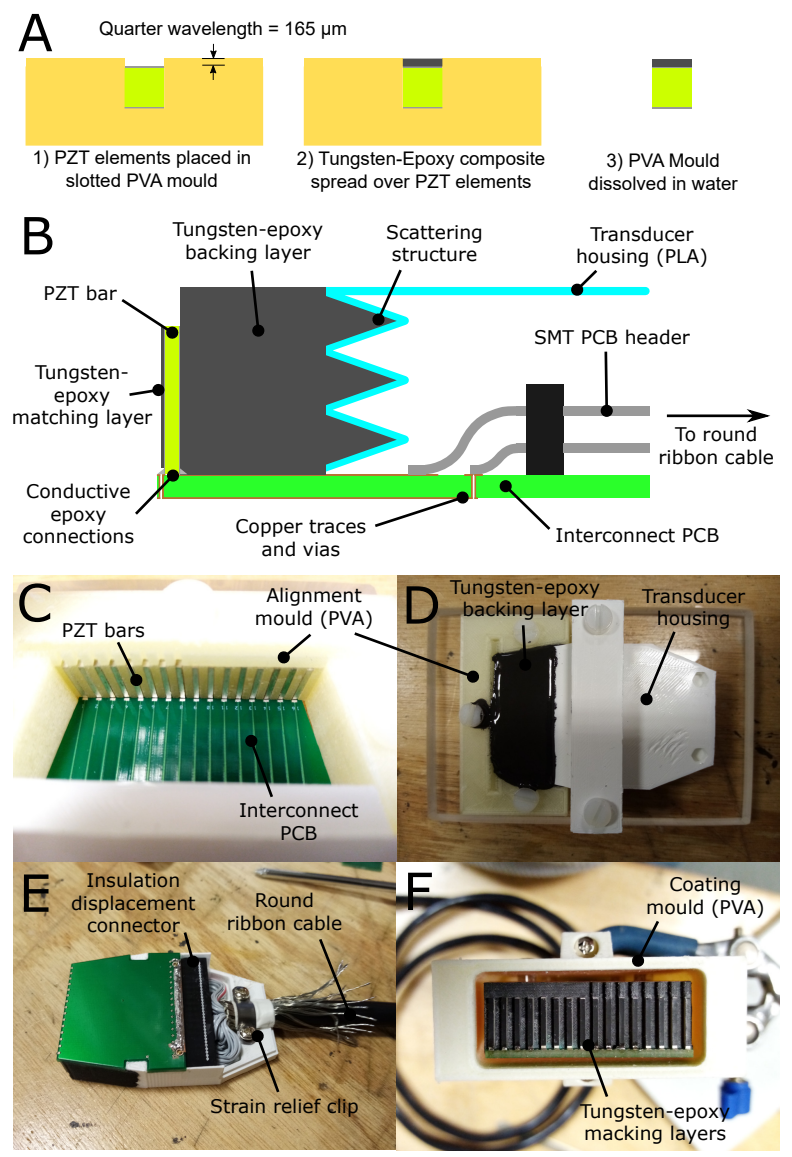

Fig. 3. A: Matching layer deposition process. B: Cross sectional transducer module schematic. C: Alignment of PZT bars with PCB and housing. D: Backing layer casting. E: Connecting to ribbon cable. F: Mould used for transducer encapsulation.

For the matching layer, $5 \mu \mathrm{m}$ tungsten powder (Tungsten Alloys, UK) and Araldite Standard epoxy resin were mixed using a silicone bowl and spatula for 10 minutes. The composite was scraped over the PZT bars using a razor blade at a $45^{\circ}$ angle, filling the quarter-wavelength space above each PZT bar, as shown in Figure 3A. The PVA mould was then dissolved in water to remove the matched PZT bars. Next, rear electrodes were terminated to pads on an interconnect PCB using 833114G conductive epoxy (MG Chemicals). Matched PZT bars were placed into another slotted 3D-printed PVA mould, to align them relative to each other and also to the PCB and transducer housing, shown in Figure 3C. The tungsten-epoxy backing layer was prepared as previously, and cast directly behind the PZT bars, shown in Figure 3D. After curing, the PVA alignment mould was dissolved in water. A 34-conductor shielded round ribbon cable $(3 \mathrm{M})$ was terminated to a header 
on the PCB using an insulation displacement connector, and secured to the transducer housing, shown in Figure 3E. Next, the front electrodes were terminated to the PCB using conductive epoxy, and the transducer was wrapped in copper foil, which was soldered to the cable braid, shown in Figure 1. The transducer was encapsulated using Araldite Standard epoxy and a 3D-printed PVA mould, shown in Figure 3F, but the PZT elements were left uncoated. Future transducers will use a polyurethane rubber with an acoustic impedance approximately equal to water, to encapsulate the PZT elements without affecting the acoustic propagation. Finally, the cable was terminated with a DL5-260P connector (ITT Cannon), which was housed in a 3D-printed PLA backshell with copper foil lining.

\section{EXPERIMENTAL EVALUATION}

\section{A. Hydrophone scans}

Hydrophone scans were performed to evaluate the far field beam shapes generated by the elements. Measurements were taken in degassed and deionised water at $22.6{ }^{\circ} \mathrm{C}$ using an $\mathrm{X}-\mathrm{Y}$ stepper motor positioning system (Precision Acoustics). All 16 channels were driven by a 3 cycle tri-state pulse at 2 $\mathrm{MHz}$ and a $50 \mathrm{~V}$ amplitude, using a Vantage-256 (Verasonics). Signals were acquired using a $200 \mathrm{~mm}$ needle hydrophone (Precision Acoustics) connected to an oscilloscope (InfiniiVision DSOX3024A, Keysight), using 128 averages in a 345 $\times 192$ point grid, with a uniform point spacing of 0.261 $\mathrm{mm}$, located $31.3 \mathrm{~mm}$ from the transducer surface, on a plane approximately perpendicular to the transducer beam axis. The hydrophone frequency response was deconvolved from the measured signals, and band-pass filtering was applied (500 $\mathrm{kHz}-5 \mathrm{MHz}$ ). Next a Tukey window was applied to the filtered signals, and these were then projected to the transducer source plane, using the angular spectrum method [11], giving a 3D pressure field. To examine the beam shape produced by a single element, the pressure time series representing the element in the source plane were isolated from the other elements, using a binary mask. For each element, the masked pressure time series were re-projected forwards into the far field to a distance of $\mathrm{z}=140 \mathrm{~mm}$, and the temporal peak at each point was extracted.

\section{B. Evaluation of inter-element skew}

The skew of the beam axis in the far field was measured for each element in the elevation and lateral planes. To define the main beam axis, a 3D linear fit to the maximum pressure at each axial position was performed. This axis was projected onto the elevation and lateral planes, and the bulk misalignment of the PZT array with respect to the hydrophone measurement plane was then removed by subtracting the mean skew from the skew of each element.

Figure 4 shows a distribution of the inter-element elevational skews centred on zero with a standard deviation of $\sigma=$ $0.41^{\circ}$, and a maximum of $0.67^{\circ}$. During image reconstruction of UST data from a ring array, it is assumed that the fanshaped beams generated by each element are coplanar. The
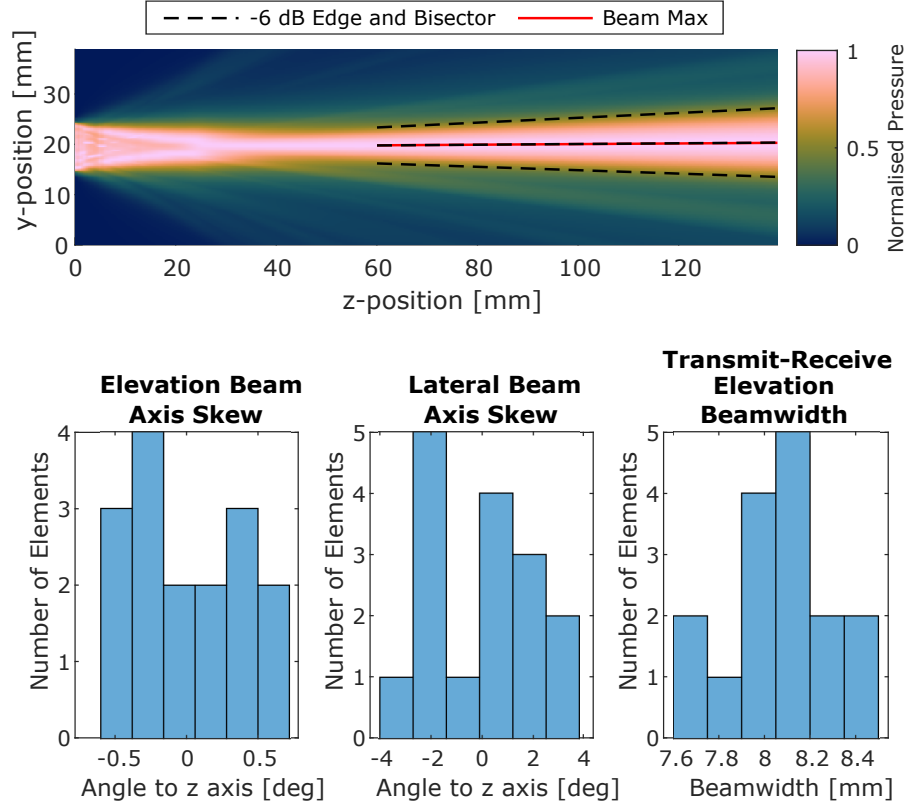

Fig. 4. Top: Temporal peak pressure distribution in the elevation plane of element 1 (normalised in the y-direction at each axial z-position). Bottom: Histograms showing the beam axis skew in the elevation and lateral planes, and the transmit-receive beamwidth

very low inter-element elevational skew seen here suggests that this system could satisfy this assumption. Figure 4 also shows that the inter-element lateral skew distribution has a standard deviation of $\sigma=2.07^{\circ}$, and a maximum of $3.62^{\circ}$, which is higher than the elevational skews. However, larger angular skews are tolerable in the lateral plane, since the beam is very divergent. The negligible misalignment shown here is significant, since it means that end users do not need to measure individual element skews or model their effect during image reconstruction. It should be noted that interelement skew has only been evaluated here within a single transducer module. To guarantee alignment throughout the entire ring, each PZT element array must be well aligned with its transducer housing, and the module housings themselves must be well aligned. These additional factors are currently being investigated.

\section{Evaluation of elevation beamwidth}

The transmit-receive beamwidths were estimated using the re-projected 3D pressure fields. For each element, the elevation plane was extracted using a maximum intensity projection, and a region between $\mathrm{z}=80 \mathrm{~mm}$ and $\mathrm{z}=140 \mathrm{~mm}$ was extracted and multiplied by a flipped copy of itself, to give the transmit-receive sensitivity. The $-6 \mathrm{~dB}$ beamwidth at the ring array centre $\mathrm{z}=110 \mathrm{~mm}$ was then calculated in the elevation direction. Figure 4 , shows a distribution of beamwidths with a mean of $\mu=8.1 \mathrm{~mm}$ and a standard deviation of $\sigma=0.23 \mathrm{~mm}$. This is slightly larger than the $7.5 \mathrm{~mm}$ beamwidth predicted previously, which suggests that the effective elevation height of the elements is shorter than their physical size of $10 \mathrm{~mm}$. This is consistent with the geometry of the matching layers, since 
the bottom $0.5 \mathrm{~mm}$ of each electrode is used for an electrical connection. The low inter-element variation in beamwidth suggests that the elements could be assumed to have identical directional responses, during image reconstruction. Additionally, the close agreement between the simulated and measured beamwidths suggests that the physical element elevation height is a good approximation to the effective radiating elevation height of each element. These factors are important, since it removes the need for end users to measure the beamwidths of every element, which could allow preliminary UST work to progress faster.

\section{Evaluation of transmit frequency response}

The transmit frequency response was measured using the same equipment setup as for the hydrophone scan, but with a transducer-hydrophone separation of $99 \mathrm{~mm}$. For each element, the hydrophone was aligned with the beam axis using the $\mathrm{X}-\mathrm{Y}$ positioning system to locate the maximum pressure in the measurement plane. Each element was driven in isolation using a single positive pulse with a $16 \mathrm{~ns}$ width, and an 80 $\mathrm{V}$ drive voltage. The signals from $66 \mathrm{~ms}$ to $70.5 \mathrm{~ms}$ were extracted and then band pass filtered $(500 \mathrm{kHz}-5 \mathrm{MHz})$. The hydrophone frequency response was deconvolved and then a Tukey window was applied, giving the time domain impulse responses shown in Figure 5.

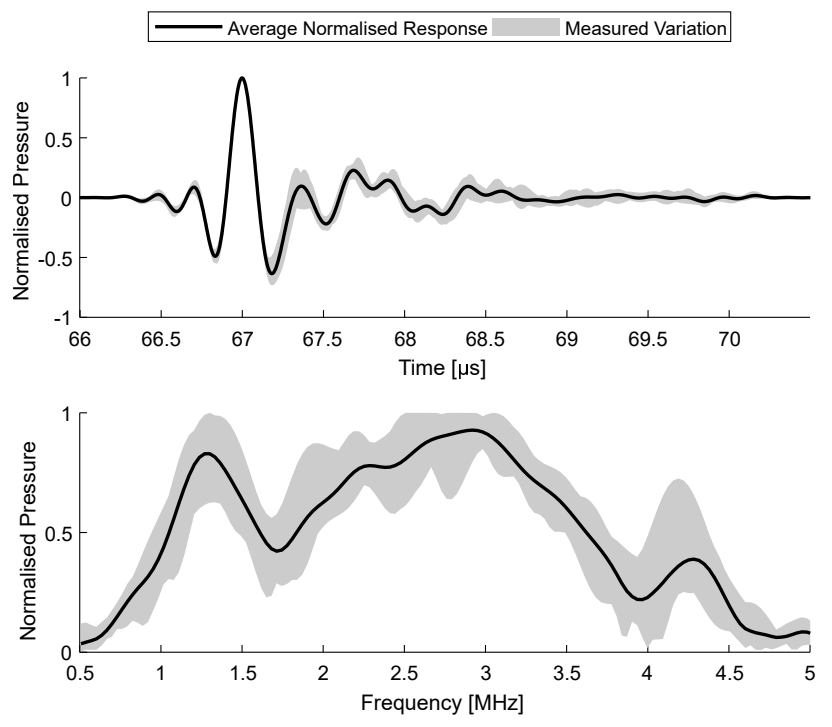

Fig. 5. Top: Impulse responses, shifted in time to align the main peaks. Bottom: Frequency responses. For both plots the black line shows the average value across all elements and the grey fill shows the range of values across all elements.

The frequency response for each element was calculated using a Fast Fourier transform, and then normalised (Figure 5). A Gaussian fit to each spectrum was used to allow cutoff frequencies to be more easily approximated. The mean centre frequency was $2.53 \mathrm{MHz} \pm 0.12 \mathrm{MHz}$ and the mean lower and upper $-6 \mathrm{~dB}$ cutoff frequencies were $1.17 \pm 0.15 \mathrm{MHz}$ and $3.87 \pm 0.16 \mathrm{MHz}$, although there may not be useful energy at all intermediate frequencies, since a local minimum occurs between $1.65 \mathrm{MHz}-1.8 \mathrm{MHz}$. After normalisation, the standard deviation in amplitude between elements is $8 \%$, averaged across all frequencies, which again represents a low interelement variation. This can also be seen in the time domain traces, where the main pulses have a highly uniform shape, and the trailing reverberations also show similar features. The low inter-element variation suggests that the frequency response of the full ring array could be modelled based on measurements from only a small subset of elements, further simplifying image reconstruction for end users.

\section{CONClusions AND Future WORK}

A prototype 16-element transducer array has been developed for use as part of a modular ring array UST system. Low inter-element variation was shown in the skew, beamwidth and frequency response, which could simplify the assumptions required during image reconstruction, and remove the need for end users to characterise individual elements. These factors could lower the barrier to entry for researchers starting preliminary UST work. Next, the full 256-element, 16-module ring array system will be fabricated and used for UST imaging. An open source hardware distribution will be released, including CAD models, files for PCB production and 3D-printed parts, and detailed documentation of manufacture procedures.

\section{REFERENCES}

[1] M. G. Marmot, D. Altman, D. Cameron, J. Dewar, S. Thompson, and M. Wilcox, "The benefits and harms of breast cancer screening: an independent review," British journal of cancer, vol. 108, no. 11, pp. 2205-2240, 2013.

[2] J. Wiskin, B. Malik, D. Borup, N. Pirshafiey, and J. Klock, "Full wave 3d inverse scattering transmission ultrasound tomography in the presence of high contrast," Scientific Reports, vol. 10, no. 1, pp. 1-14, 2020.

[3] D. Sarno, C. Baker, M. Hodnett, and B. Zeqiri, "Phase-insensitive ultrasound computed tomography for acoustic attenuation imaging of breast phantoms," in Medical Imaging 2021: Ultrasonic Imaging and Tomography, vol. 11602. International Society for Optics and Photonics, 2021, p. 116020T.

[4] M. Roberts, E. Martin, B. Cox, and B. Treeby, "Progress towards an open-source, low-cost ultrasound tomography research system," Proceedings of International Workshop on Medical Ultrasound Tomography 2019, pp. 53-66.

[5] B. Malik, R. Terry, J. Wiskin, and M. Lenox, "Quantitative transmission ultrasound tomography: Imaging and performance characteristics," Medical physics, vol. 45, no. 7, pp. 3063-3075, 2018.

[6] C. Li, N. Duric, P. Littrup, and L. Huang, "In vivo breast soundspeed imaging with ultrasound tomography," Ultrasound in medicine \& biology, vol. 35, no. 10, pp. 1615-1628, 2009.

[7] F. Lucka, M. Pérez-Liva, B. E. Treeby, and B. T. Cox, "High resolution 3d ultrasonic breast imaging by time-domain full waveform inversion," arXiv preprint arXiv:2102.00755, 2021.

[8] B. E. Treeby, J. Budisky, E. S. Wise, J. Jaros, and B. Cox, "Rapid calculation of acoustic fields from arbitrary continuous-wave sources," The Journal of the Acoustical Society of America, vol. 143, no. 1, pp. 529-537, 2018.

[9] E. S. Wise, B. Cox, J. Jaros, and B. E. Treeby, "Representing arbitrary acoustic source and sensor distributions in fourier collocation methods," The Journal of the Acoustical Society of America, vol. 146, no. 1, pp. 278-288, 2019.

[10] M. G. Grewe, T. Gururaja, T. R. Shrout, and R. E. Newnham, "Acoustic properties of particle/polymer composites for ultrasonic transducer backing applications," IEEE transactions on ultrasonics, ferroelectrics, and frequency control, vol. 37, no. 6, pp. 506-514, 1990.

[11] X. Zeng and R. J. McGough, "Evaluation of the angular spectrum approach for simulations of near-field pressures," The Journal of the Acoustical Society of America, vol. 123, no. 1, pp. 68-76, 2008. 